\title{
Caracterização micrográfica de próteses dentais de alumina infiltrada com vidro de lantânio e sua correlação com a resistência à flexão por três pontos
}

\author{
OGASAWARA, T. ${ }^{\mathrm{I}}$; SILVA, P.T.G. ${ }^{\mathrm{I}}$; SILVA, R.C. ${ }^{\mathrm{I}}$; PINTO, V.B.B. ${ }^{\mathrm{I}}$ \\ ${ }^{\mathrm{I}}$ Laboratório de Cerâmicas Dentais - PEMM/COPPE/UFRJ CP: 68505 - 21941-972, Rio de Janeiro, RJ. \\ e-mail: ogasawat@metalmat.ufrj.br, ptagasi@mealmat.ufrj.br, \\ raqcs@terra.com.br, bemfica@metalmat.ufrj.br
}

RESUMO

Estudou-se a infiltração (em diferentes condições) do vidro de lantânio em alumina parcialmente sinterizada e a sua relação com a resistência à flexão. As barras de In-Ceram Alumina, fraturadas em flexão por 3 pontos, foram lixadas, polidas e caracterizadas por microscopias ótica e eletrônica de varredura (MEV). Foram usadas 10 barras para cada condição operacional. Resultados: (a) A MEV confirma que os núcleos de barras In-Ceram Alumina (sinterizados a $1120^{\circ} \mathrm{C}$ durante $2 \mathrm{~h}$ ) infiltrados a $1100^{\circ} \mathrm{C}$ durante $6 \mathrm{~h}$ contém menos vidro de lantânio do que aqueles das barras infiltradas durante $6 \mathrm{~h}$ a $1110^{\circ} \mathrm{C}$; (b) A micrografia ótica confirma que os núcleos de barras In-Ceram Alumina (sinterizada a $1120^{\circ} \mathrm{C}$ durante $2 \mathrm{~h}$ ) infiltrada durante $6 \mathrm{~h}$ (ou $2 \mathrm{~h}$ ) nas temperaturas de $1100^{\circ} \mathrm{C}, 1100^{\circ} \mathrm{C}, 1120^{\circ} \mathrm{C}$ e $1130^{\circ} \mathrm{C}$ não se diferem notavelmente; (c) A MEV mostra que a microestrutura interna de barras In-Ceram Alumina infiltradas com vidro de lantânio a $1120^{\circ} \mathrm{C}$ e $1130^{\circ} \mathrm{C}$ é complexa.

Palavras-chaves: Cerâmica dental, alumina infiltrada com vidro, análise ceramográfica.

\section{Micrographic characterization of lanthanum glass infiltrated alumina dental prosthesis and its correlation to the three-point flexural strength ABSTRACT}

The infiltration of partially sintered alumina (under different conditions) by lanthanum aluminosilicate glass and its relation to the three-point flexural strength were studied. The In-Ceram alumina bars, fractured by flexural test, were ground by sand-paper, polished, and characterized by optical and scanning electron microscopy (SEM). A total of 10 bars have been used for each operational condition. Results: a) SEM confirmed that the inner region of the In-Ceram ${ }^{\circledR}$ alumina bars (sintered at $1120^{\circ} \mathrm{C}$ for $2 \mathrm{~h}$ ) infiltrated at $1100^{\circ} \mathrm{C}$ during $6 \mathrm{~h}$ contain less lanthanum glass than that of alumina bar infiltrated by glass at $1110^{\circ} \mathrm{C}$ for $6 \mathrm{~h}$; (b) The optical micrography confirmed that the cores of the In-Ceram ${ }^{\circledR}$ Alumina bars (sintered at $1120^{\circ} \mathrm{C}$ for 2h) infiltrated during $6 \mathrm{~h}$ (or $2 \mathrm{~h}$ ) at temperatures of $1100^{\circ} \mathrm{C}, 1100^{\circ} \mathrm{C}, 1120^{\circ} \mathrm{C}$ and $1130^{\circ} \mathrm{C}$ do not differ significantly each other; (c) The SEM showed that the internal microstructure of the In-Ceram ${ }^{\circledR}$ Alumina bars infiltrated with lanthanum glass at $1120^{\circ} \mathrm{C}$ and $1130^{\circ} \mathrm{C}$ is complex.

Key words: Dental ceramics, glass-infiltrated alumina, ceramographic analysis, three-point flexural strength.

\section{INTRODUÇÃO}

In-Ceram ${ }^{\circledR}$ Alumina, inventada em 1987 por TYZBLAT [1] como reportado por WILDGNOOSE et al [2] é um compósito cerâmico, de alta resistência mecânica, empregado na fabricação de coroas dentárias e prótese parcial fixa anterior de até três elementos [ $\underline{3}-\underline{3}]$. A alumina processada pelo uso da técnica de colagem em barbotina (pelo pincelamento de uma barbotina contendo $65 \%$ de alumina sobre a superfície de um molde de plástico) e sinterizada em baixas temperaturas (aquecimento da alumina até $1100^{\circ} \mathrm{C}$ onde permanece durante $2 \mathrm{~h}$ ) dá origem a uma subestrutura porosa (18 MPa de resistência à flexão) capaz de suportar, criticamente, o manuseio e a usinagem [6]. Este precursor é a seguir submetido à infiltração de um vidro de alumino-silicato de lantânio, objetivando sua densificação e fortalecimento [6]; o alcance deste objetivo depende do pleno molhamento da alumina parcialmente sinterizada pelo vidro líquido [7]. A diferença do 
coeficiente de expansão térmica entre a alumina e o vidro conduz a uma tensão compressiva no vidro na interface alumina-vidro, o que aumenta a resistência mecânica do material [ $\underline{2}, \underline{8}]$. De fato, o produto infiltrado com vidro passa a ter uma resistência à flexão de $300 \mathrm{MPa}$ a $600 \mathrm{MPa}$ [9], valor substancialmente maior do aquele de outros materiais cerâmicos usados anteriormente. Após isto, o compósito vidro-alumina limpo (tendo removido o excesso de vidro) recebe um revestimento de porcelana de cobertura (a $900^{\circ} \mathrm{C}$, aproximadamente) para conferir forma, função e estética semelhantes àqueles do dente natural [10].

O sistema In-Ceram ${ }^{\circledR}$ Alumina ocupa uma posição de destaque em função da sua fiel adaptação marginal em coroas unitárias terminadas com ombros arredondados (como um resultado do alto teor de alumina, com tamanhos de partícula na faixa de 0,5 a 3,5 $\mu \mathrm{m}$, com tamanho médio igual a 2,85 $\mu \mathrm{m}$ [11]). A isto se associa uma baixa retração linear (de apenas $0,3 \%$ na sua sinterização a $1120^{\circ} \mathrm{C}$ ), compensada pela expansão do molde de plástico especial na sua cura $(0,4 \%)$, o que resulta em praticamente nenhuma contração global [6] . O resultado é um processo de fabricação simples [12-14], que leva a subestrutura bifásica de fases contínuas mutuamente embutidas, a uma adaptação precisa [11] ao dente preparado.

Próteses parciais fixas integralmente cerâmicas (PPFs) devem ter altura e largura suficientes para suportar os esforços mastigatórios, o que em In-Ceram ${ }^{\circledR}$ Alumina significa conectores tendo $4 \mathrm{~mm}$ na direção gengival-oclusal e $3 \mathrm{~mm}$ na direção bucal-lingual [5] .

O presente trabalho teve por objetivo estudar a correlação entre a microestrutura do subestrutura dental cerâmica e a sua resistência à flexão por 3 pontos.

\section{MATERIAIS E MÉTODOS}

Os materiais empregados no estudo foram: placas acrílicas, discos de carborundum para corte das placas acrílicas, polivinilsiloxano (Elite Double), Gesso para In-Ceram ${ }^{\circledR}$ Alumina, pó de alumina, solvente aquoso, aditivo para In-Ceram ${ }^{\circledR}$ Alumina, pó de vidro de alumino-silicato de lantânio, pasta de alumina para polimento, pasta de diamante de 1 a $4 \mu \mathrm{m}$ para polimento.

Os equipamentos usados foram agitador por vibração VH Softline, misturador ultrassônico Vitasonic II, Forno Inceramat 3, Forno Vita Vacumat 40, micromotor de baixa rotação (W\&H MF-Percta) para usinagem manual, broca de diamante de granulação grosseira (EVE-DIABOND DB-15g), Jateador Trijet Easy-Blast (Bego), máquina universal de ensaios mecânicos EMIC (DL-1000), Microscópio Eletrônico de Varredura (JEOL, JSM-64860 LV).

Sessenta corpos de prova de In-Ceram ${ }^{\circledR}$ Alumina foram fabricados (com $4 \mathrm{~mm}$ de largura, $25 \mathrm{~mm}$ de comprimento e $1,4 \mathrm{~mm}$ de espessura) em estado parcialmente sinterizado de acordo com a norma ISO (ISO $6871 ; 1995(\mathrm{E}))$, que especifica largura $=4 \pm 0,25 \mathrm{~mm}$, comprimento mínimo $=20 \mathrm{~mm}$ e espessura $=$ $1,2 \pm 0,2 \mathrm{~mm}$. Estes corpos de prova foram preparados usando 2 modelos de acrílico, como descrito abaixo.

O primeiro modelo acrílico possuindo uma seção reta retangular e medindo $2 \mathrm{~mm}$ x $5 \mathrm{~mm} \times 25 \mathrm{~mm}$ foi usado para produzir um molde de polivinilsiloxano. Neste molde foi vazado gesso comercial para o sistema In-Ceram ${ }^{\circledR}$ Alumina, após manuseio (ajudado com espátula) de acordo com a instrução do fabricante, que consistiu no uso de $20 \mathrm{~g}$ de pó de plástico e 4,6ml de água bi-destilada; o gesso foi vazado no molde de polivinilsiloxano sob ação de um vibrador de gesso (VH softline), para evitar a retenção de bolha. O processo de cristalização do gesso completou-se em $2 \mathrm{~h}$, após o que foi removido do molde e lixado a seco (com lixa d'água) e verificado quando às suas dimensões devidas (2mm x $5 \mathrm{~mm}$ x $25 \mathrm{~mm})$ usando um calibre.

O segundo modelo de acrílico foi construído com dimensões $4 \mathrm{~mm}$ x $5 \mathrm{~mm}$ x $25 \mathrm{~mm}$ (que são as dimensões do espaço ocupado pelo modelo de gesso acima citado [ $2 \mathrm{~mm}$ x $5 \mathrm{~mm}$ x $25 \mathrm{~mm}$ ], acrescido do volume a ser ocupado pelo futuro corpo de prova de In-Ceram ${ }^{\circledR}$ Alumina $[1,2 \pm 0,2 \mathrm{~mm}$ x $4 \pm 0,25 \mathrm{~mm} \times 25 \mathrm{~mm}]$ mais um excesso de alumina a fim de obter um perfeito corpo de prova por meio de usinagem do corpo de prova parcialmente sinterizado, previamente feito por colagem em barbotina. Este modelo também serviu para obter um segundo molde de polivinilsiloxano cujo papel foi a fabricação do substrato de gesso sobre o qual a barbotina de In-Ceram ${ }^{\mathbb{B}}$ Alumina foi colada para alcançar a borda superior do molde de polivinisiloxano.

Uma vez completada a colagem da barbotina sobre o molde de gesso, a barra-dupla (barra verde de alumina + barra de gesso) foi removida do molde de polivinilsiloxano e submetida a lento aquecimento e sinterização no forno Vita Inceramat ${ }^{\circledR} 3$. O programa de aquecimento recomendado pelo fabricante consistiu no seguinte: pré-aquecimento até $120^{\circ} \mathrm{C}$ numa taxa de $20^{\circ} \mathrm{C} / \mathrm{h}$, seguido por um segundo aquecimento numa taxa de $500^{\circ} \mathrm{C} / \mathrm{h}$ de $120^{\circ} \mathrm{C}$ até $1120^{\circ} \mathrm{C}$, onde o material cerâmico foi mantido durante $2 \mathrm{~h}$, antes do resfriamento no interior do próprio forno.

As 60 barras sinterizadas foram divididas em sub-grupos de 10 barras cada para a próxima etapa que consistiu de infiltração de vidro de alumino-silicato de lantânio. A duração desta infiltração foi de $6 \mathrm{~h}$ a $1100^{\circ} \mathrm{C}$ (Grupo I), $1110^{\circ} \mathrm{C}$ (Grupo II), $1120^{\circ} \mathrm{C}$ (Grupo III) e $1130^{\circ} \mathrm{C}$ (Grupo IV). A das barras do grupo V foram infiltradas a $1110^{\circ} \mathrm{C}$ em $2 \mathrm{~h}$. As 10 restantes amostras sinterizadas serviram para exames de 
microscopia eletrônica de varredura no estado parcialmente sinterizado, sem infiltração de vidro, bem como repetição de testes de infiltração confirmatórios.

Cada amostra de alumina parcialmente sinterizada recebeu cobertura de pó de vidro de lantânio misturado com água na posição como colocado sobre uma placa de platina $(95 \% \mathrm{Pt}+5 \% \mathrm{Au})$. O histórico térmico da infiltração de vidro (realizado no forno Inceramt 3) foi o seguinte: introdução no forno préaquecido a $200^{\circ} \mathrm{C}$, aquecimento até $1110^{\circ} \mathrm{C}\left(1100^{\circ} \mathrm{C}, 1120^{\circ} \mathrm{C}\right.$ ou $\left.1130^{\circ} \mathrm{C}\right)$ em aproximadamente 30 minutos, manutenção na temperatura de infiltração durante $6 \mathrm{~h}$ (Grupos I ao IV) ou 2h (Grupo V).

Após a infiltração de vidro, o excesso de vidro existente em cada amostra foi removido por meio de broca de diamante numa peça de mão com micromotor (W\&H MF-Perfecta) operando a $30000 \mathrm{rpm}$, a fim de alcançar as dimensões de $1,4 \mathrm{~mm} \times 4 \mathrm{~mm} \times 25 \mathrm{~mm}$. A etapa seguinte foi o jateamento com partículas de alumina de $80 \mu \mathrm{m}$ e pressão de 2,5 bar. As barras limpas lixadas a úmido em lixas de carbeto de silício de granulação $220,360,600$ e 1200, seguido de polimento final com pasta de diamante de $2 \mathrm{a} 4 \mu \mathrm{m}$.

Após o acabamento superficial, as barras de alumina foram submetidas a 2 queimas de controle em forno Vita Vacumat 40 , mediante aquecimento direto até $600^{\circ} \mathrm{C}$ e aquecimento subseqüente até $960^{\circ} \mathrm{C}$ em 5 minutos $\left(72^{\circ} \mathrm{C} /\right.$ minuto), manutenção durante 10 minutos a $960^{\circ} \mathrm{C}$ e resfriamento ao ar livre.

O ensaio de flexãol por 3 pontos foi feito numa máquina universal de ensaios mecânicos EMIC DL1000. A distância entre os apoios (de acordo com a norma ISO 6872, 1995(E) para cerâmicas dentais) foi de $12 \mathrm{~mm}$ e a velocidade de flexão foi de $0,5 \mathrm{~mm} / \mathrm{min}$. De acordo com a norma ASTM C1161-90, o teste de flexão deve ser feito observando as seguintes condições: (a) comprimento dos apoios deveria ser igual a 3 vezes o comprimento das barras testadas; (b) diâmetro dos apoios cilindros de (a) deve ser igual a 1,5 vezes a espessura da barra testada; (c) o vão entre apoios ("spam") deve ser controlado com precisão igual a $\pm 0,10 \mathrm{~mm}$. A cabeça aplicadora da carga flexural deveria situar no meio do vão ("spam") com uma tolerância de $\pm 0,10 \mathrm{~mm}$.

A superfície de fratura de cada uma das barras submetidas ao ensaio flexão foi lixada e polida (com alumina e pasta de diamante) para exame ceramográfico (ótico e eletrônico de varredura). A determinação do tamanho médio do grão de alumina nas barras In-Ceram ${ }^{\circledR}$ Alumina foi feita medindo-se grão a grão, numa impressão tamanho A4 de uma apropriada micrografia eletrônica de varredura da supra-referida seção polida da amostra fraturada: cada grão foi medido em 4 direções distintas ("vertical", "horizontal" e nas duas bissetrizes em relação a estas duas direções referenciais). Em cada impressão micrográfica foi determinado o número total de grãos de alumina, seu diâmetro médio e o correspondente desvio padrão. A porcentagem de fases foi determinada usando uma impressão tamanho A3 de uma apropriada micrografia eletrônica de varredura: (a) efetuou-se a separação das 3 fases (poros, grãos de alumina e vidro infiltrado) por corte (usando bisturi e tesoura); (b) os recortes de cada fase foram pesados numa balança analítica de 4 casas decimais; (c) este peso de (b) constituiu a integração da área parcial da fase numa amostragem de superfície de corte da barra e proporcional a $\mathrm{l}_{\mathrm{i}}^{2}$ (onde $\mathrm{l}_{\mathrm{i}}$ é a aresta do cubo representativo do volume parcial da fase no volume total da cerâmica). Portanto, o volume de uma fase é proporcional a (Peso parcial da fase) $)^{3 / 2}$. A soma dos volumes parciais das fases dá o volume total da cerâmica, $\mathrm{V}_{\text {total }}=\operatorname{Soma}\left(\mathrm{V}_{\mathrm{i}}\right)$. Assim a porcentagem de vidro infiltrado $=\left(\mathrm{V}_{\text {vidro }} / \mathrm{V}_{\text {total }}\right) \times 100$, uma relação semelhante aplicando-se para as outras fases (poros e grãos de alumina). Como o resultado final de interesse é porcentagem, não há problema nenhum no uso de peso como representante de $l_{i}^{2}$ e de seu uso na geração de $V_{i}=(\text { peso da fase } i)^{3 / 2}$

\section{RESULTADOS}

A tabela 1 apresenta os resultados de resistência à flexão juntamente com aqueles da porcentagem de vidro infiltrado. Pode-se ver que a infiltração numa temperatura menor do que a recomendada pelo fabricante $\left(1100^{\circ} \mathrm{C}\right.$ em vez de $\left.1110^{\circ} \mathrm{C}\right)$ conduziu a uma porcentagem menor de vidro infiltrado no produto final, enquanto que a infiltração numa temperatura superior àquela recomendada pelo fabricante $\left(1130^{\circ} \mathrm{C} \mathrm{em}\right.$ vez de $1110^{\circ} \mathrm{C}$ ) conduziu também a uma menor porcentagem de vidro no produto final. Isto parece indicar que $1110^{\circ} \mathrm{C}$ recomendada pelo fabricante representa uma condição ótima cujas razões não são conhecidas na literatura.

A tabela 2 mostra os resultados de tamanho médio do grão de alumina nas amostras micrográficas dos 5 grupos de amostras. Considerando os tamanhos médios do grão de alumina de todos os grupos, bem como os seus desvios-padrões, conclui-se que pequenas diferenças na temperatura de infiltração do vidro não acarretaram diferenças nos tamanhos de grão de alumina do produto final, ou seja, desprezível efeito da sinterização complementar. 
Tabela 1: Resistência à flexão por 3 pontos e porcentagem de vidro infiltrado.

\begin{tabular}{|c|c|c|c|}
\hline \multirow[b]{2}{*}{ Grupos de amostras (GR) } & \multicolumn{2}{|c|}{ Resistência à flexão (MPa) } & \multirow[b]{2}{*}{$\%$ de Vidro Infiltrado } \\
\hline & Média & Desvio Padrão & \\
\hline I ou GR(I) & 580,75 & 99,21 & 5,67 \\
\hline II ou GR(II) & 605,47 & 75,17 & $9,57^{*}$ \\
\hline III ou GR(III) & 579,34 & 92,14 & ND \\
\hline IV ou GR(IV) & 590,40 & 139,31 & 8,31 \\
\hline V ou GR(V) & 410,16 & 178,19 & ND \\
\hline
\end{tabular}

Tabela 2: Tamanho médio dos grãos de alumina nos produtos finais dos 5 Grupos de amostras do estudo.

\begin{tabular}{|l|l|l|}
\hline \multirow{2}{*}{ Grupos de amostras (GR) } & \multicolumn{2}{|l|}{ Tamanho de grão de alumina ( $\boldsymbol{\mu m})$} \\
\cline { 2 - 3 } & Médio & Desvio Padrão \\
\hline I ou GR(I) & 1,62 & 0,99 \\
\hline II ou GR(II) & 1,76 & 0,84 \\
\hline III ou GR(III) & ND & ND \\
\hline IV ou GR(IV) & $1,608^{*}$ & $0,88^{* *}$ \\
\hline V ou GR(V) & ND & ND \\
\hline
\end{tabular}

$\mathrm{ND}=$ não disponível. *(média das médias de 5 amostras micrográficas do mesmo Grupo). **(desvio padrão médio dos desvios padrões de 5 amostras micrográficas do mesmo grupo).

A Figura 1 ilustra as micrografias eletrônicas de varredura de cada um dos três Grupos de amostras examinados. Estas micrografias corroboram visualmente a essência do que dizem os números da Tabela 2.

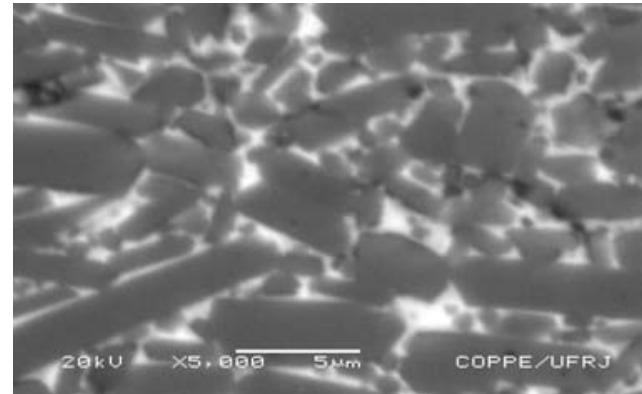

(a) GR(I) barra 3

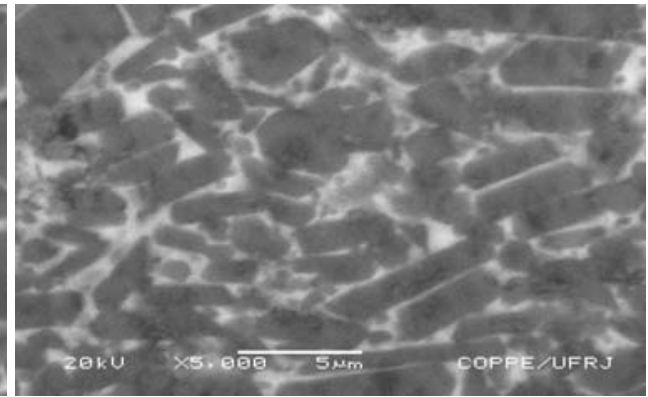

(b) GR(II) barra 1 .

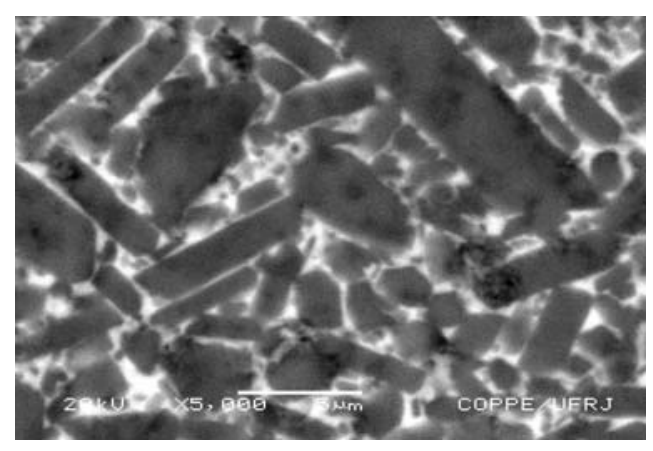

(c) GR(IV) barra 1

Figura 1: Micrografias eletrônicas de varredura de barras do: (a) Grupo I, (b) Grupo II e (c) Grupo IV.

A Figura 2 mostra micrografias óticas dos núcleos (região central da seção de fratura, em seu estado polido) das amostras dos 5 Grupos estudados, não revelando nenhuma diferença flagrante de microestrutura ou de porosidade entre elas. 


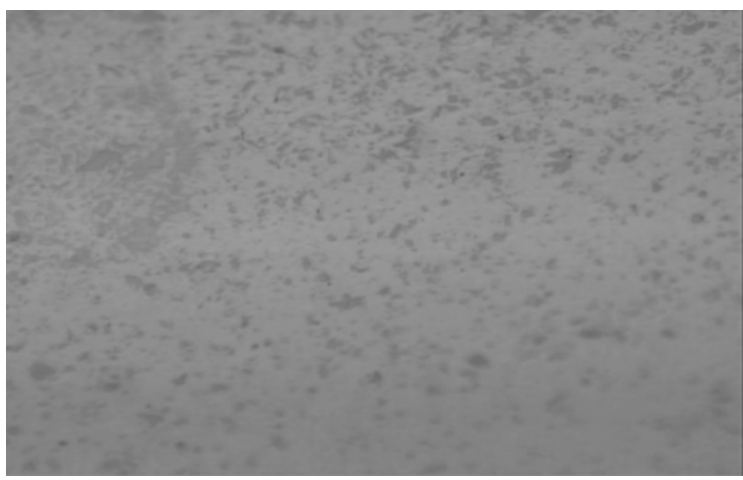

(a) GR(I) barra 1-m

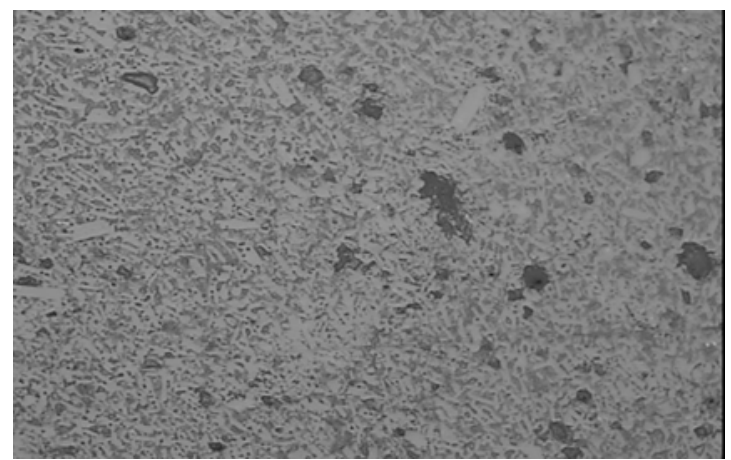

(c) GR(III) barra 3-m

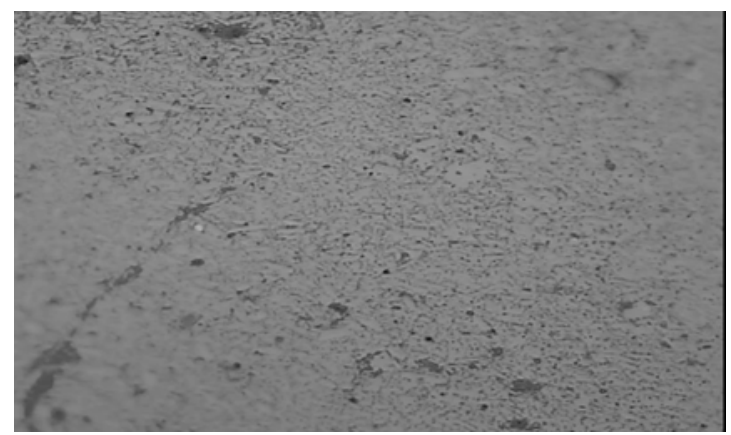

(d) GR(V) barra 5-m

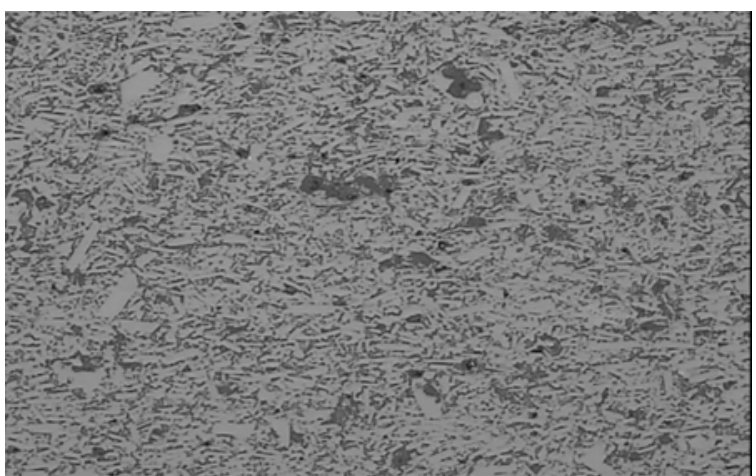

(b) GR(II) barra 2-m

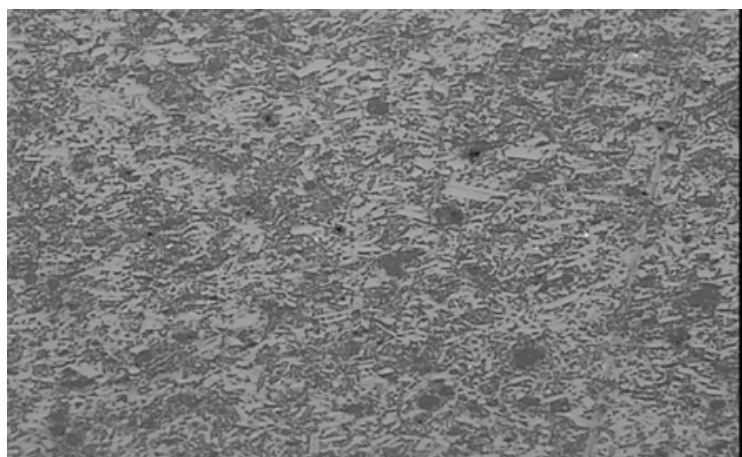

(d) GR(IV) barra 4-m

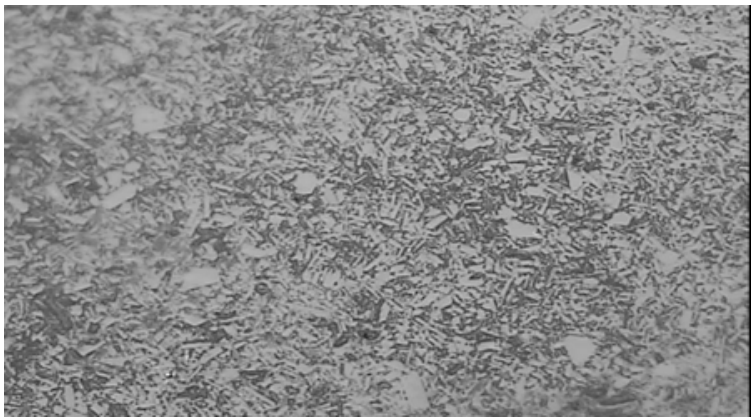

(f) GR(V) barra 5-s

Figura 2: Micrografias óticas das seções de fratura ( polidas) de amostras dos Grupos I a V. ( $\mathrm{m}=$ meio ou núcleo, $\mathrm{s}=$ borda sul).

\section{DISCUSSÃO}

Conforme detalhadamente discutido na pesquisa de dissertação de mestrado de Raquel [15], o módulo de Weibull resultou igual a 6,4, 8,73, 8,57, 3,86 e 2,25, com coeficientes de correlação de 97\%, 98\%, 91\%, 95\% e 97\%, respectivamente, para os Grupos I a V no que tange aos resultados de resistência flexural por 3 pontos. O aumento do valor do módulo de Weibull $(\mathrm{m})$ significa menor dispersão dos resultados e maior confiabilidade dos mesmos $[\underline{16}, \underline{17}]$. No presente trabalho, o módulo de Weibull do Grupo I é menor do que aquele do Grupo II (infiltração como recomendado pelo fabricante), o que pode corresponder a uma condição ótima de infiltração (encontrada em pesquisa do fabricante) e provavelmente decorrente do comportamento reológico do vidro de alumino-silicato de lantânio em função da temperatura de infiltração. Cabe lembrar que todo vidro é metaestável e tenderá a cristalizar-se (com enorme aumento da viscosidade, que pode perfeitamente prejudicar a efetiva introdução do vidro nos poros da infra-estrutura de alumina parcialmente sinterizada).

Naturalmente, o aumento da temperatura de infiltração do vidro (acima daquela da sinterização parcial prévia, como nos casos dos Grupos III e IV) pode implicar numa sinterização complementar paralela 
da alumina à infiltração do vidro, dificultando a eficiência desta última. É claro que os melhores resultados de penetração capilar de vidro líquido em estruturas cerâmicas porosas ocorre quando os poros são adequadamente grandes [18]. Aqui também pensou-se nesta hipótese, mas os resultados micrográficos obtidos não corroboram nesta direção. É claro que, pesquisa mais pormenorizada, confrontando a distribuição dos poros superficiais das barras de alumina infiltradas com vidro com o que acontece no interior da mesma barra deveria ser ainda examinada (uma vez que, no processo de infiltração, a superfície da barra estará sempre mais quente do que o interior, por simples razões de transferência de calor). Este trabalho não chegou a ser realizado aqui por simples falta de oportunidade temporal.

Todavia, necessariamente, a variação do comportamento reológico do infiltrante vidro de aluminosilicato de lantânio terá que ser examinado para explicar por que uma temperatura de infiltração de $1110^{\circ} \mathrm{C}($ conforme recomendado pelo fabricante) oferece os melhores resultados de infiltração e de resistência à flexão. Infelizmene a temperatura de amolecimento do vidro de alumino-siliato de lantânio não é encontrada na literatura publicada disponível e, assim, necessita ser determinada no próximo trabalho experimental. Muito provavelmente existe um compromisso entre a variação da viscosidade do vidro em relação à temperatura de infiltração e o efeito desta última no fechamento dos poros da alumina .

\section{CONCLUSÕES}

a) Pequenas diferenças de temperatura de infiltração de vidro de lantânio (acima de $1110^{\circ} \mathrm{C}$, como recomendado pelo fabricante) não afetam a resistência à flexão do casquete de alumina infiltrada com o referido vidro porque o efeito desta variação de temperatura sobre a quantidade de vidro infiltrado no produto final não é significativo; temperatura de infiltração menor do que $1110^{\circ} \mathrm{C}$, efetivamente reduz a quantidade de vidro infiltrado.

b) O exame micrográfico, após lixa e polimento, das seções de fratura dos corpos de prova de teste de resistência à flexão não revelou nenhum aumento notável de tamanho de grão da alumina com o aumento da temperatura de infiltração de vidro acima de $1110^{\circ} \mathrm{C}$ recomendado pelo fabricante.

c) Um exame mais acurado da alteração morfológica da camada superficial das amostras infiltradas pelo vidro de lantânio (em contraste com as características do interior das mesmas amostras) deveria ser realizado, mas um exame da variação do comportamento reológico do vidro de lantânio com função da temperatura acima de $1110^{\circ} \mathrm{C}$ deveria ser necessariamente realizada no sentido de obter um melhor entendimento dos resultados de resistência à flexão obtidos no presente trabalho.

\section{AGRADECIMENTOS}

Os autores agradecem a todos que direta ou indiretamente contribuíram para a concretização deste trabalho, em particular ao CNPq e à FAPERJ pelo apoio financeiro (Pronex de Cerâmicas Dentais, Proc. E26/171.204/2003).

\section{BIBLIOGRAFIA}

[1] TYSBKAT, M., "Process for the preparation of a dental prosthesis by slight solid phase fritting of a metal oxide based infrastructure", United States Patent n. 4, 772, 436, USA, September 20, 1988.

[2] WILDGNOOSE, G.D., JOHNSON, A., WINSTANLEY, R.B., "Glass/ ceramic/ refractory techniques, their development and introduction into dentistry: A historical review", The Journal of Prosthetic Dentistry, v. 91, n. 2, pp. 136-43, 2004.

[3] Mc LAREN, E.A., WHITE, S.N., "Survival of in-ceram crowns in a private practice: A prospective clinical trial", The Journal of Prosthetic Dentistry, v. 83, n. 2, pp. 216-22, 2000.

[4] RAIGRODSKI, A.J., "Contemporary materials and technologies for all-ceramic fixed partial dentures: A review of the literature", The Journal of Prosthetic Dentistry, v. 92, pp. 557-562, 2004.

[5] RAIGRODSKI, A.J., CHICHE, G.J., "The safety and efficacy of anterior ceramic fixed partial dentures: A review of the literature", The Journal of Prosthetic Dentistry, v. 86, n. 5, pp. 520-525, 2001.

[6] XIAO-PING, L., JIE-MO, T., YUN-LONG, Z., LING, W., "Strength and fracture toughness of MgOmodified glass infiltrated alumina for CAD/CAM”, Dental Materials, v.18, pp. 216-20, 2002. 
[7] RIZKALLA, A.S., JONES, D.W., "Mechanical properties of commercial high strength ceramic core materials", Dental Materials, v. 20, n. 3, pp. 207-12, 2004.

[8] WOOD, D.J., et al. "Preliminary investigation of a novel retentive system for hydrofluoric and etchresistant dental ceramics", The Journal of Prosthetic Dentistry, v. 78, n. 3, pp. 275-80, Sep, 1997.

[9] DE MIRANDA, C.C., "In-ceram alumina system”, Revista Brasileira de Prótese Clínica \& Laboratorial, ano 1, n. 2, 1999.

[10] APHOLT, W., BINDL, A., LÜTHY, H., MÖRMANN, W.H., "Flexural strength of cerec 2 machined and jointed in ceram-alumina and in ceram-zirconia bars", Dental Materials, v. 17, n. 3, pp. 260$267,2001$.

[11] LEE, D.Y., KIM, D.J., KIM, B-Y., SONG Y-S., "Effect of alumina particle size and fracture toughness of alumina-glass composites prepared by melt infiltration", Materials Science and Engineering A, v. A341, pp. 98-105, 2003.

[12] KERN, M., THOMPSON, V.P., "Sandblasting and silica coating of a glass infiltrated alumina ceramic: Volume loss, morphology, and changes in the surface composition", The Journal of Prosthetic Dentistry, v. 71, n. 5, pp. 453-61, 1994.

[13] KERN, M., THOMPSON, V.P., "Bonding to glass infiltrated alumina ceramics: Adhesive methods and their durability", The Journal of Prosthetic Dentistry, v. 73, pp. 240-9, 1995.

[14] MADANI, M., "Effects of surface treatments on shear bond strengths between a resin cement and alumina core", The Journal of Prosthetic Dentistry, v. 83, n. 6, pp. 644-7, 2000.

[15] SILVA, R.C., Influência do processamento de infiltração na resistência à flexão e na microestrutura do compósito alumina-vidro de lantânio, Dissertação de Mestrado em Engenharia Metalúrgica e de Materiais, COPPE/UFRJ, xiv, 101p., março/2006

[16] SULLIVAN, J.D., LAUZON, P.H., "Experimental Probability Estimators for Weibull Plots”, Journal of Materials Science Letters, v. 5, pp. 1245-1247, 1986.

[17] GÖHRING, T.N., GALLO, L., LÜTHY, H., "Effect of water storage, thermocycling, the incorporation and site of placement of glass-fibers on the flexural strength of veneering composite", Dental Materials, v. 21, pp. 761-772, 2005.

[18] LEE, D.Y., KIM, D.J., KIM, B-Y., SONG, Y-S., "Effect of alumina particle size and distribution on infiltration rate and fracture toughness of alumina-glass composites prepared by melt infiltration", Materials Science and Engineering A, v. A341, pp. 98-105, 2003. 